\title{
HYPERSURFACE SECTIONS OF TORIC SINGULARITIES
}

\author{
By Hiroyasu Tsuchinashi
}

\section{Introduction}

As is well-known, we can obtain much information about hypersurface singularities $\{f=0\}$ in $\boldsymbol{C}^{n+1}$ by the Newton polyhedra $\Gamma_{+}(f) \subset \boldsymbol{R}^{n+1}$ of the defining equations $f$. (For instance, see [5] and [11].) In this paper, we define the Newton polyhedra also for hypersurface sections $(X, x)$ of any toric singularity $(Y, y)$ and show that a part of the results in [11] are valid. On the other hand, as we see in the last of $\S 2$ and in $\S 3$, we obtain as $(X, x)$ many singularities, a part of which are not complete intersections. For instance, 2-dimensional cusp singularities with multiplicities greater than 4 and a 3-dimensional singularity with a resolution whose exceptional set is an Enriques surface. Moreover, in the case that the ambient space $Y$ has only an isolated singularity, these singularities $(X, x)$ are obviously smoothable. Hence we can obtain examples of smoothable cusp singularities (see $\S 3$ ). In this paper, we are mainly concerned about singularities $(X, x)$ with the plurigenera $\delta_{m}(X, x)$ which are not greater than 1 and at least one of which is equal to 1 . (For the definition of plurigenera, see [11].) We call such singularities, periodically elliptic singularities, following Ishii [2].

In Section 1, we recall some facts about toric singularities, necessary in this paper.

In Section 2, we show a sufficient condition on the Newton polyhedra of defining equations $f$ of $X$, under which $(X, x)$ are periodically elliptic singularities and give some examples.

In Section 3, we show a sufficient condition on a 3-dimensional non-terminal Gorenstein toric singularity $(Y, y)$, under which hyperplane sections $(X, x)$ of $(Y, y)$ are simple elliptic singularities or cusp singularities. We can determine the multiplicities of these singularities.

In Section 4, we show that if $H^{1}\left(X \backslash\{x\}, i * \Theta_{Y}\right)=0$ and $\operatorname{dim} X \geqq 3$, then we can concretely construct a locally semiuniversal family of deformations of $(X, x)$ and that any small deformation of $(X, x)$ is also a hypersurface section of $Y$, where $i: X \subset Y$ lis [the inclusion map and $\Theta_{Y}$ is the tangent sheaf of $Y$. The above condition is satisfied, if $Y$ is a quotient of $C^{n+1}$, by torus actions.

We use the notation and the terminology in [4] freely.

I would like to thank Professor M. Tomari who pointed out me the facts

Received August 21, 1989 ; revised November 14, 1990. 
that hypersurface sections $(X, x)$ of toric singularities $(Y, y)$ are Cohen-Macaulay and that $(X, x)$ are smoothable, if $(Y, y)$ is an isolated singularity.

\section{$\S 1$. Toric singularities}

Let $N$ be a free $Z$-module of rank $n+1$ and let $N_{R}=N \otimes_{z} R$. Let $M=$ $\operatorname{Hom}(N, \boldsymbol{Z})$ be the $\boldsymbol{Z}$-module dual to $N$ with the canonical pairing $\langle\rangle:, M \times N \rightarrow \boldsymbol{Z}$. Let $\sigma=\boldsymbol{R}_{\geq 0} u_{1}+\boldsymbol{R}_{\geq 0} u_{2}+\cdots+\boldsymbol{R}_{\geq 0} u_{s}$ be an $(n+1)$-dimensional strongly convex rational polyhedral cone in $N_{\boldsymbol{R}}$. Here we may assume that $\boldsymbol{R}_{\geq 0} u_{\imath}$ are 1-dimensional faces of $\sigma$, for $i=1$ through $s$. Let $Y$ be the complex space associated to $\operatorname{Spec}\left(\boldsymbol{C}\left[M \cap \sigma^{*}\right]\right)$ and let $\boldsymbol{e}(v): Y \rightarrow \boldsymbol{C}$ be the natural extension to $Y$ of the character $v \otimes 1_{C^{\times}}: T_{N} \rightarrow C^{\times}$for each $v$ in $M \cap \sigma^{*}$, where $\sigma^{*}:=\left\{v \in M_{R} \mid\langle v, u\rangle \geqq 0\right.$ for all $u \in \sigma \backslash\{0\}\}$ is the dual cone of $\sigma$ and $T_{N}=\operatorname{Spec}(\boldsymbol{C}[M])\left(\cong\left(\boldsymbol{C}^{\times}\right)^{n+1}\right)$. Then any holomorphic function $f$ on a neighborhood $U$ of $y=\operatorname{orb}(\sigma)$ is expressed as the series :

$$
f=\sum_{v \in \sigma * \cap M} c_{v} e(v) .
$$

Hence we can define the Newton polyhedron $\Gamma_{+}(f)$ and the Newton boundary $\Gamma(f)$ of $f$ in the same way as in the case of $Y=C^{n+1}$. More precisely, $\Gamma_{+}(f)$ is the convex hull of $\cup_{c_{v} \neq 0} v+\sigma^{*}$ and $\Gamma(f)$ is the union of the compact faces of $\Gamma_{+}(f)$. Let $D=D_{1}+D_{2}+\cdots+D_{s}$, where $D_{\imath}$ is the closure of $\operatorname{orb}\left(\boldsymbol{R}_{\geq 0} u_{\imath}\right)$. Here we note that $Y \backslash D=T_{N}$ and that $Y$ is a Cohen-Macaulay space by [4, Corollary 3.9]. Let $\left\{v_{1}, v_{2}, \cdots, v_{n+1}\right\}$ be a basis of $M$ and let $w_{i}=\boldsymbol{e}\left(v_{i}\right)$ for $i=1$ through $n+1$. Then $\left(w_{1}, w_{2}, \cdots, w_{n+1}\right)$ is a global coordinate of $T_{N}$. Let $\nu=\left(d w_{1} / w_{1}\right) \wedge$ $\left(d w_{2} / w_{2}\right) \wedge \cdots \wedge\left(d w_{n+1} / w_{n+1}\right)$. Then $\nu$ is a nowhere vanishing holomorphic $(n+1)$ form on $T_{N}$ whose natural extension to $Y$ has poles of order 1 along $D$.

Definition 1.1. $(Y, y)$ is said to be $r$-Gorenstein, if there exists a nowhere vanishing holomorphic $r$-ple $(n+1)$-form on $U \backslash \operatorname{Sing}(U)$ for an open neighborhood $U$ of $y$, where $\operatorname{Sing}(U)$ is the singular locus of $U$.

Since $(Y, y)$ is a Cohen-Macaulay singularity, $(Y, y)$ is Gorenstein, if it is 1-Gorenstein.

Proposition 1.2. ([6, the footnote of p294]) $(Y, y)$ is $r$-Gorenstein, if and only if there exists an element $v_{0}$ in $M_{Q}$ such that $r v_{0} \in M$ and that $\left\langle v_{0}, u_{\imath}\right\rangle=1$ for $i=1$ through $s$, where we assume that $u_{1}, u_{2}, \cdots$ and $u_{s}$ are primitive elements in $N$. (Here we note that the above $v_{0}$ is uniquely determined by $\sigma$, if it exists.)

Proof. Let $v_{0}$ be an element in $M_{\boldsymbol{Q}}$ satisfying the above condition. Then $\theta:=e\left(r v_{0}\right) \nu^{r}$ is a nowhere vanishing holomorphic $r$-ple $(n+1)$-form on $Y \backslash \operatorname{Sing}(Y)$, because $\boldsymbol{e}\left(r v_{0}\right)$ has zeros of order $\left\langle r v_{0}, u_{2}\right\rangle=r$ only along $D$. Conversely, assume that $(Y, y)$ is $r$-Gorenstein, i.e., there exists a nowhere vanishing holomorphic $r$-ple $(n+1)$-form $\theta$ on $U \backslash \operatorname{Sing}(U)$ for an open neighborhood $U$ of $y$. Then $f:=\theta / \nu^{r}$ is a holomorphic function on $U \backslash \operatorname{Sing}(U)$ which does not vanisn on 
$T_{N} \cap U$ and whose vanishing order at $D_{\imath}$ is equal to $r$. Since the codimension of $\operatorname{Sing}(Y)$ is greater than $1, f$ is extended to $U$, by [1, Chapter II, Corollary 3.12]. Hence $f$ ie expressed as the series $\sum_{v \in(\sigma * \backslash(0)) \cap M} c_{v} e(v)$. Suppose that $\Gamma_{+}(f)$ has a compact face $\Delta$ with $\operatorname{dim} \Delta \geqq 1$. Then there exist a primitive element $u_{0}$ in $\operatorname{Int}(\sigma) \cap N$ and a positive integer $t$ such that $\left\langle v, u_{0}\right\rangle=t$ (resp. $>t$ ) for any element $v$ in $\Delta$ (resp. $\left.\Gamma_{+}(f) \backslash \Delta\right)$. Let $Y_{0}$ be the complex space associated to $\operatorname{Spec}\left(\boldsymbol{C}\left[\left(\boldsymbol{R}_{\geq 0} u_{0}\right)^{*} \cap M\right]\right)\left(\cong \boldsymbol{C} \times\left(\boldsymbol{C}^{\times}\right)^{n}\right)$ and let $D_{0}=\operatorname{orb}\left(\boldsymbol{R}_{\geq 0} u_{0}\right)$. Then we have a holomorphic map $\pi: Y_{0} \rightarrow Y$ such that $\pi_{\mid T_{N}}=i d$ and that $\pi^{-1}(y)=D_{0}$, because $\boldsymbol{R}_{>0} u_{0} \subset \operatorname{Int}(\sigma)$. Take a basis $\left\{v_{1}^{\prime}, v_{2}^{\prime}, \cdots, v_{n+1}^{\prime}\right\}$ of $M$ so that $\left\langle v_{1}^{\prime}, u_{0}\right\rangle=1$ and that $\left\langle v_{i}^{\prime}, u_{0}\right\rangle=0$ for $i=2$ through $n+1$. Let $z_{2}=\boldsymbol{e}\left(v_{i}^{\prime}\right)$ for $i=1$ through $n+1$. Then $D_{0}=\left\{z_{1}=0\right\}$ and $f=z_{1}^{t} g_{0}+z_{1}^{t+1} g_{1}+\cdots+z_{1}^{t+2} g_{i}+\cdots$ on $U \cap \boldsymbol{T}_{N}$, where $g_{\imath}=\Sigma_{v \in L_{2}}$ $c_{v} \boldsymbol{e}\left(v-(t+i) v_{1}^{\prime}\right)$ and $L_{\imath}=\left\{v \in \Gamma_{+}(f) \cap M \mid\left\langle v, u_{0}\right\rangle=t+i\right\}$. Here we note that $g_{\imath}$ are polynomials with variables $z_{2}, \cdots, z_{n+1}$ and that $g_{0}=\sum_{v \in \Delta \cap M} c_{v} e\left(v-t v_{1}^{\prime}\right)$ is not a monomial, because the cardinal number of $\left\{v \in \Delta \cap \backslash M \mid c_{v} \neq 0\right\}$ is greater than 1 . Hence $\left\{y^{\prime} \in U \cap T_{N} \mid\left(g_{0}+z_{1} g_{1}+\cdots\right)\left(y^{\prime}\right)=0\right\} \neq \varnothing$, because $Y \backslash D_{0}=T_{N}$. Then $f$ must vanish at a point of $U \cap T_{N}$, a contradiction. Therefore, any compact face of $\Gamma_{+}(f)$ is a point. This implies that $\Gamma(f)$ consists of only one point $v_{0}^{\prime}$. Hence $\Gamma_{+}(f)=v_{0}^{\prime}+\sigma^{*}$. Therefore, $\left\langle v_{0}^{\prime}, u_{\imath}\right\rangle \leqq\left\langle v, u_{i}\right\rangle$ for any element $v$ in $\Gamma_{+}(f) \cap M$ and for $i=1$ through $n+1$. Since the vanishing order of $f$ at $D_{\imath}$ is $r$, we have $\left\langle v_{0}^{\prime}, u_{\imath}\right\rangle=r$. Hence the point $v_{0}=(1 / r) v_{0}^{\prime}$ satisfies the condition of the proposition.

q.e.d.

Remark. If $N=\boldsymbol{Z}^{n+1}$ and $\boldsymbol{\sigma}=\left(\boldsymbol{R}_{z_{0}}\right)^{n+1}$, then $Y$ is isomorphic to $\boldsymbol{C}^{n+1}$ and the point $y$ corresponds to the origin. Clearly $v_{0}=(1,1, \cdots, 1)$ satisfies the condition of the above proposition, if we identify $M$ with $N$, by the canonical inner product.

\section{$\S 2$. Hypersurface sections}

Let $f$ be an element of the maximal ideal $\mathfrak{m}_{Y, y}$ of $Y$ at $y$, let $X=\{f=0\}$ and let $x=y$. Throughout the rest of this paper, we assume that $n=\operatorname{dim} X \geqq 2$, that $X$ is irreducible reduced, that $(X, x)$ is an isolated singularity and that $X \cap \operatorname{Sing}(Y)=\{x\}$. By [1, Chapter I, Proposition 1.6 (ii) and Corollary 4.4], we have:

Proposition 2.1. $(X, x)$ is a Cohen-Macaulay and normal singularity.

Assume that $f=\Sigma_{v \in(\sigma * \backslash(0)) \cap M} c_{v} \boldsymbol{e}(v)$ is non-degenerate, i.e.,

$$
\partial f_{\Delta} / \partial w_{1}=\partial f_{\Delta} / \partial w_{2}=\cdots=\partial f_{\Delta} / \partial w_{n+1}=0
$$

has no solutions in $T_{N}=Y \backslash D\left(\cong\left(C^{\times}\right)^{n+1}\right)$, for each face $\Delta$ of $\Gamma(f)$, where $f_{\Delta}=$ $\sum_{v \in \Delta \cap M} c_{v} \boldsymbol{e}(v)$ and $\left(w_{1}, w_{2}, \cdots, w_{n+1}\right)$ is a global coodinate of $T_{N}$.

Theorem 2.2. Assume that $(Y, y)$ is $r$-Gorenstein, (that $(Y, y)$ is not $r^{\prime}$ Gorenstein for $\left.1 \leqq r^{\prime}<r\right)$ and let $v_{0}$ be the element satisfying the condition of 
Proposition 1.2. Then $(X, x)$ is $r$-Gorenstein. Moreover, if $v_{0}$ is on $\Gamma(f)$, then

$$
\delta_{m}(X, x)=\left\{\begin{array}{l}
1 \text { for } m \equiv 0 \bmod r \\
0 \text { for } m \neq 0 \bmod r .
\end{array}\right.
$$

Conversely, if $\max \left\{\delta_{m}(X, x) \mid m \in Z, m>0\right\}=1$, then $v_{0}$ is on $\Gamma(f)$. (See [11], for the definition of $\delta_{m}(X, x)$.)

For the proof, we need some preparations. For $u \in \sigma$, let $d(u)=\min \{\langle v, u\rangle \mid$ $\left.v \in \Gamma_{+}(f)\right\}$ and let $\Delta(u)=\left\{v \in \Gamma_{+}(f) \mid\langle v, u\rangle=d(u)\right\}$. For a face $\Delta$ of $\Gamma_{+}(f)$, let $\Delta^{*}=\{u \in \sigma \mid \Delta(u) \supset \Delta\}$. Then $\Gamma^{*}(f):=\left\{\Delta^{*} \mid \Delta\right.$ is a face of $\left.\Gamma_{+}(f)\right\} \cup\{0\}$ is an r.p.p. decomposition of $N_{R}$ with $\left|\Gamma^{*}(f)\right|\left(:=U_{\Delta^{*} \in \Gamma *(f)} \Delta^{*}\right)=\sigma$. Let $\Sigma^{*}$ be a subdivision of $\Gamma *(f)$ consisting of non-singular cones and let $\tilde{Y}=T_{N} \operatorname{emb}\left(\Sigma^{*}\right)$. Then we have a resolution $\Pi: \tilde{Y} \rightarrow Y$ of $Y$. Let $\tilde{X}$ be the proper transformation of $X$ under $\Pi$ and let $E=\tilde{X} \cap \Pi^{-1}(x)$. Then $\pi\left(:=\Pi_{\mid \tilde{X}}\right): \tilde{X} \rightarrow X$ is a resolution of $X$ whose exceptional set is $E$. Assume that $u$ is a primitive element in $N$ and that $\boldsymbol{R}_{\geq 0} u$ is a 1 -dimensional cone in $\Sigma^{*}$ with $\operatorname{dim} \Delta(u) \geqq 1$. Then we denote by $E(u)$ the closure of $\operatorname{orb}\left(\boldsymbol{R}_{\geq 0} u\right) \cap E(\neq \phi)$. Recall that $\theta:=\boldsymbol{e}\left(r v_{0}\right) \nu^{r}$ is a nowhere vanishing $r$-ple $(n+1)$-form on $Y \backslash \operatorname{Sing}(Y)$. Let $\omega=\operatorname{Res}\left(\theta / f^{r}\right)$, i.e., $\omega=$ $g_{1 X \cap U}\left(d w_{1} \wedge \cdots \wedge d w_{n}\right)^{r}$ on $X \cap U$, if $\theta$ is expressed as $g\left(d f \wedge d w_{1} \wedge \cdots \wedge d w_{n}\right)^{r}$ on an open set $U$ of $Y$.

LEMMA 2.3. $\pi * \omega^{l}$ has zeros of order $\operatorname{lr}\left(\left\langle v_{0}, u\right\rangle-1-d(u)\right)$ along $E(u)$.

Proof. The lemma follows from the fact that $\boldsymbol{e}\left(r v_{0}\right), \nu^{r}$ and $\left(\pi^{*} f\right)^{r}$ have zeros of order $r\left\langle v_{0}, u\right\rangle,-r$ and $r d(u)$, respectively, along $\operatorname{orb}\left(\boldsymbol{R}_{\geq 0} u\right)$. q.e.d.

Proof of Theorem 2.2. Since $\omega$ is a nowhere vanishing holomorphic $r$-ple $n$-form on $X \backslash\{x\}$, we see that $(X, x)$ is $r$-Gorenstein. Assume that $v_{0}$ is on $\Gamma(f)$. Then $\left\langle v_{0}, u\right\rangle \geqq d(u)$ for any $u$ in $\operatorname{Int}(\sigma) \cap N$. Hence the nowhere vanishing holomorphic $l r$-ple $n$-form $\pi^{*} \omega^{l}$ has poles of order at most $l r$ along each irreducible component of the exceptional set $E$, by Lemma 2.3. On the other hand, $\Gamma_{+}(f)$ has a compact face $\Delta_{0}$ containing $v_{0}$ with $\operatorname{dim} \Delta_{0} \geqq 1$. Otherwise, $\Gamma_{+}(f)=v_{0}+\sigma^{*}$ and hence $f=\boldsymbol{e}\left(v_{0}\right) g$ for a holomorphic function $g$ on $Y$. Then since $\left[\boldsymbol{e}\left(v_{0}\right)\right]=r D$, we get a contradiction to the assumption that $X$ is irreducible. Hence we can take a subdivision $\Sigma^{*}$ of $\Gamma^{*}(f)$ so that $\Delta\left(u_{0}\right)=\Delta_{0}$ for a 1-dimensional cone $\Delta^{*}=\boldsymbol{R}_{\geq 0} u_{0}$ in $\Sigma^{*}$. Then $u_{0} \in \operatorname{Int}(\sigma), \operatorname{orb}\left(\Delta^{*}\right) \cap \tilde{X} \neq \varnothing$ and $\left\langle v_{0}, u_{0}\right\rangle=$ $d\left(u_{0}\right)$. Hence $\pi^{*} \omega^{l}$ has poles of order $l r$ along the irreducible component $E\left(u_{0}\right)$ of $E$. Therefore, $\delta_{l r}(X, x)=1$. Next, assume that $m \neq 0 \bmod r$ and let $\eta$ be an element in $H^{0}\left(X \backslash\{x\}, \mathcal{O}_{X}\left(m K_{X}\right)\right)$. In the following, we show that $\eta$ is in $L^{2 / m}(X \backslash\{x\})$. We note that $r v_{0}$ is a primitive element in $M$. Otherwise, $(Y, y)$ is $r^{\prime}$-Gorenstein for a positive integer $r^{\prime}<r$. Hence we can take $n$ elements $v_{1}, v_{2}, \cdots$ and $v_{n}$ in $M$ so that $\left\{r v_{0}, v_{1}, \cdots, v_{n}\right\}$ is a basis of $M$. Let $w_{0}=\boldsymbol{e}\left(r v_{0}\right)$ and let $w_{2}=\boldsymbol{e}\left(v_{i}\right)$ for $i=1$ through $n$. Then $\left(w_{0}, w_{1}, \cdots, w_{n}\right)$ is a global coordinate of $T_{N}$. Let $M^{\prime}=M+Z v_{0}$ and let $N^{\prime}=\left\{u \in N \mid\left\langle v^{\prime}, u\right\rangle \in Z\right.$ for any $\left.v^{\prime} \in M^{\prime}\right\} \quad(=\{u$ 
$\left.\left.\in N \mid\left\langle v_{0}, u\right\rangle \in Z\right\}\right)$. Then the inclusion $N^{\prime} \rightarrow N$ induces a holomorphic map $\varphi: Y^{\prime}$ $\rightarrow Y$, where $Y^{\prime}$ is the complex space associated to $\operatorname{Spec}\left(\boldsymbol{C}\left[M^{\prime} \cap \sigma^{*}\right]\right)$. Since $\left\{v_{0}, v_{1}, \cdots, v_{n}\right\}$ is a basis of $M^{\prime},\left(z_{0}, z_{1}, \cdots, z_{n}\right)$ is a global coordinate of $T_{N^{\prime}}=$ $\operatorname{Spec}\left(\boldsymbol{C}\left[M^{\prime}\right]\right)$, where $z_{\imath}=\boldsymbol{e}\left(v_{i}\right)$ for $i=0$ through $n$. Clearly, $\varphi^{*} w_{0}=\left(z_{0}\right)^{r}$ and $\varphi^{*} w_{\imath}=z_{\imath}$ for $i=1$ through $n$. Hence $\varphi$ is the quotient map under the group $\langle t\rangle$ generated by the element $t=(\xi, 1, \cdots, 1)$ in $T_{N^{\prime}}$, where $\xi$ is a primitive $r$-th root of 1. Moreover, $\varphi$ is unramified over $Y \backslash \operatorname{Sing}(Y)$, because $\theta:=w_{0}\left(\left(d w_{0} / w_{0}\right)\right.$ $\left.\wedge\left(d w_{1} / w_{1}\right) \wedge \cdots \wedge\left(d w_{n} / w_{n}\right)\right)^{r}\left(\right.$ resp. $\left.\theta^{\prime}:=z_{0}\left(d z_{0} / z_{0}\right) \wedge\left(d z_{1} / z_{1}\right) \wedge \cdots \wedge\left(d z_{n} / z_{n}\right)\right)$ is a nowhere vanishing holomorphic $r$-ple $(n+1)$-form on $Y \backslash \operatorname{Sing}(Y)$ (resp. $(n+1)$ form on $\left.Y^{\prime} \backslash \operatorname{Sing}\left(Y^{\prime}\right)\right)$ and $\varphi^{*} \theta=\left(r \theta^{\prime}\right)^{r}$. Hence Sing $\left(X^{\prime}\right)=\left\{x^{\prime}\right\}$, where $X^{\prime}:=$ $\varphi^{-1}(X)$ and $x^{\prime}:=\varphi^{-1}(x)$. Let $\omega^{\prime}=\operatorname{Res}\left(\theta^{\prime} / \varphi^{*} f\right)$. Then $\omega^{\prime}$ is a nowhere vanishing holomorphic $n$-form on $X^{\prime} \backslash\left\{x^{\prime}\right\}$ with $t^{*} \omega^{\prime}=\xi \omega^{\prime}$, because $t^{*} z_{0}=\xi z_{0}$ and $t^{*}\left(d z_{i} / z_{\imath}\right)$ $=d z_{i} / z_{2}$ for $i=0$ through $n$. Hence $\varphi^{*} \eta=g\left(\omega^{\prime}\right)^{m}$ for a holomorphic function $g$ on $X^{\prime}$. Since $t^{*}\left(\varphi^{*} \eta\right)=\varphi^{*} \eta$ and $t^{*}\left(g\left(\omega^{\prime}\right)^{m}\right)=t^{*} g \xi^{m}\left(\omega^{\prime}\right)^{m}$, we have $t^{*} g=\xi^{-m} g$. Since $\xi^{-m} \neq 1$, we have $g\left(x^{\prime}\right)=0$. Hence $\varphi^{*} \eta=g\left(\omega^{\prime}\right)^{m} \in \mathcal{L}^{2 / m}\left(X^{\prime} \backslash\left\{x^{\prime}\right\}\right)$, because $\left(\pi^{\prime}\right)^{*} \omega^{\prime} \in H^{0}\left(\tilde{X}^{\prime}, \mathcal{O}\left(K_{\tilde{X}^{\prime}}+E^{\prime}\right)\right)$, for any resolution $\pi^{\prime}:\left(\tilde{X}^{\prime}, E^{\prime}\right) \rightarrow\left(X^{\prime}, x^{\prime}\right)$ of $\left(X^{\prime}, x^{\prime}\right)$. Therefore, $\eta \in \mathcal{L}^{2 / m}(X \backslash\{x\})$. Thus we conclude that $\delta_{m}(X, x)=0$. Finally, note that if $v_{0} \notin \Gamma_{+}(f)$ (resp. $\in \operatorname{Int}\left(\Gamma_{+}(f)\right)$ ), then $\delta_{m}(X, x) \geqq 2$ for certain positive integers $m$ (resp. $=0$ for all positive integers $m$ ), by Lemma 2.3 and that if $v_{0} \in$ $\partial \Gamma_{+}(f) \backslash \Gamma(f)$, then $(X, x)$ is not isolated. Thus we obtain the last assertion of the theorem.

q.e.d.

We can obtain a system of defining equations of $X$ from those of $Y$ and $f$.

Proposition 2.4. If $f \notin \mathfrak{m}_{Y, y}^{2}$ (resp. $\left.f \in \mathfrak{m}_{Y, y}^{2}\right)$, then $\operatorname{dim} \mathfrak{m}_{X, x} / \mathfrak{m}_{X, x}^{2}=$ $\operatorname{dim} \mathfrak{m}_{Y, y} / \mathfrak{m}_{Y, y}^{2}-1$ (resp. $\left.\operatorname{dim} \mathfrak{m}_{Y, y} / \mathfrak{m}_{Y, y}^{2}\right)$.

Proof. We have the following exact sequence.

$$
0 \longrightarrow f \cdot \mathcal{O}_{Y, y} /\left(f \cdot \mathcal{O}_{Y, y} \cap \mathfrak{m}_{Y, y}^{2}\right) \longrightarrow \mathfrak{m}_{Y, y} / \mathfrak{m}_{Y, y}^{2} \longrightarrow \mathfrak{m}_{X, x} / \mathfrak{m}_{X, x}^{2} \longrightarrow 0 .
$$

We easily see that $\operatorname{dim} f \cdot \mathcal{O}_{Y, y} /\left(f \cdot \mathcal{O}_{Y, y} \cap \mathfrak{m}_{Y, y}^{2}\right)=1$ or 0 , according as $f \notin \mathfrak{m}_{Y, y}^{2}$ or $f \in \mathfrak{m}_{Y, y}^{2}$.

q.e.d.

Assume that $\sigma^{*} \cap M$ is generated by $m$ elements $v_{1}, v_{2}, \cdots, v_{m}$ and let $z_{\imath}=$ $\boldsymbol{e}\left(v_{i}\right)$, for $i=1$ through $m$. Then we have the embedding $i: Y \ni p \mapsto\left(z_{1}(p), z_{2}(p)\right.$, $\left.\cdots, z_{m}(p)\right) \in \boldsymbol{C}^{m}$. Assume that $i(Y)$ is defined by $g_{1}(z)=g_{2}(z)=\cdots=g_{t}(z)=0$, where $z=\left(z_{1}, z_{2}, \cdots, z_{m}\right)$. If $f \in \mathfrak{m}_{Y, y}^{2}$, then $X=\{f=0\}$ is isomorphic to the subvariety in $\boldsymbol{C}^{m}$ defined by $\tilde{f}(z)=g_{1}(z)=\cdots=g_{t}(z)=0$, where $\tilde{f}(z)$ is a holomorphic function on $\boldsymbol{C}^{m}$ with $i^{*} \tilde{f}=f$. Next, assume that we can express $\tilde{f}(z)=$ $z_{1}-h\left(z_{2}, \cdots, z_{m}\right)$. Hence $f \notin \mathfrak{m}_{Y, y}^{2}$. Then $X$ is isomorphic to the subvariety in $\boldsymbol{C}^{m-1}$ defined by $g_{1}^{\prime}(w)=g_{2}^{\prime}(w)=\cdots=g_{t}^{\prime}(w)=0$, where $w=\left(z_{2}, \cdots, z_{m}\right)$ and $g_{i}^{\prime}(w)=$ $g_{i}\left(h(w), z_{2}, \cdots, z_{m}\right)$.

Example 1. Let $n=2$, let $\left\{u_{1}, u_{2}, u_{3}\right\}$ be a basis of $N$ and let $\left\{v_{1}, v_{2}, v_{3}\right\}$ be the basis of $M$ dual to $\left\{u_{1}, u_{2}, u_{3}\right\}$. Let $\sigma=\boldsymbol{R}_{\geq 0}\left(u_{1}+u_{3}\right)+\boldsymbol{R}_{\geq 0}\left(u_{1}+u_{2}+u_{3}\right)+$ 

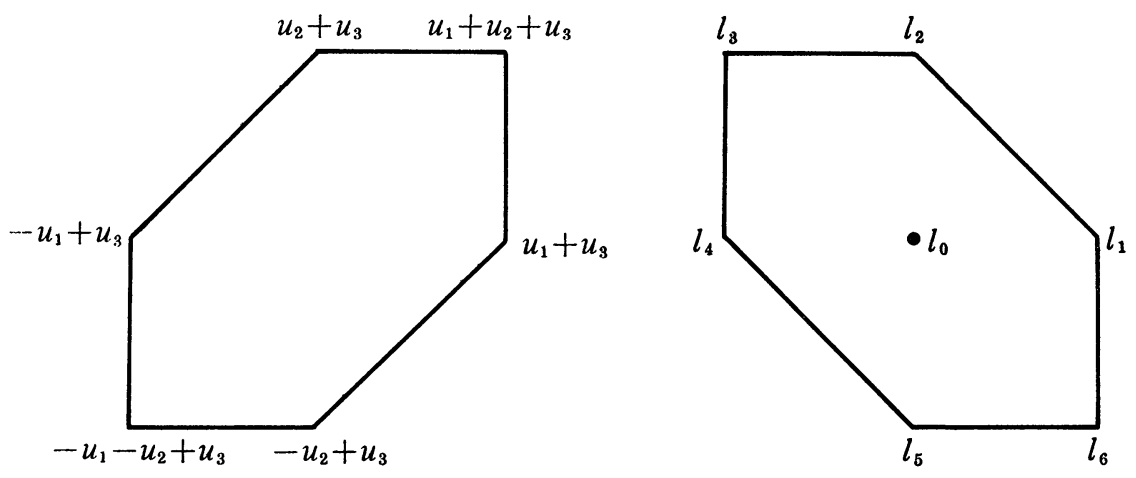

Figure 1.

$\boldsymbol{R}_{\geqq 0}\left(u_{2}+u_{3}\right)+\boldsymbol{R}_{\geqq 0}\left(-u_{1}+u_{3}\right)+\boldsymbol{R}_{\geq 0}\left(-u_{1}-u_{2}+u_{3}\right)+\boldsymbol{R}_{\geqq 0}\left(-u_{2}+u_{3}\right)$. Then $(Y, y)$ is Gorenstein and $v_{0}=v_{3}$ satisfies the condition of Proposition 1.2. We see that $\sigma^{*} \cap M$ is generated by $l_{0}=v_{3}, l_{1}=v_{1}+v_{3}, l_{2}=v_{2}+v_{3}, l_{3}=-v_{1}+v_{2}+v_{3}, l_{4}=-v_{1}+v_{3}$, $l_{5}=-v_{2}+v_{3}$ and $l_{6}=v_{1}-v_{2}+v_{3}$. (See Figure 1.) Hence $Y$ is isomorphic to the subvariety in $C^{7}$ defined by the equations (1) $z_{0} z_{1}-z_{6} z_{2}=z_{0} z_{2}-z_{1} z_{3}=z_{0} z_{3}-z_{2} z_{4}=$ $z_{0} z_{4}-z_{3} z_{5}=z_{0} z_{5}-z_{4} z_{6}=z_{0} z_{6}-z_{5} z_{1}=z_{0}^{2}-z_{1} z_{4}=z_{0}^{2}-z_{2} z_{5}=z_{0}^{2}-z_{3} z_{6}=0$, where $z_{2}=e\left(l_{2}\right)$, for $i=0$ throuh 6 . Let $f=z_{0}-z_{1}^{2}-z_{2}^{2}-\cdots-z_{6}^{2}$. Then $(X, x)$ is a cusp singularity with a resolution $\pi:(\tilde{X}, E) \rightarrow(X, x)$ such that the exceptional set $E$ is a cycle of six rational curves whose self-intersection numbers are all -3 . Since $f \notin$ $\mathfrak{m}_{Y, y}^{2}$, we see that $X$ is isomorphic to the subvariety in $C^{6}$ defined by the equations obtained from the above equations (1), replacing $z_{0}$ by $z_{1}^{2}+z_{2}^{2}+\cdots+z_{6}^{2}$.

Example 2. Let $n,\left\{u_{1}, u_{2}, u_{3}\right\}$ and $\left\{v_{1}, v_{2}, v_{3}\right\}$ be the same as in Example 1 . Let $\sigma=\boldsymbol{R}_{\geq 0}\left(u_{1}+2 u_{3}\right)+\boldsymbol{R}_{\geq 0}\left(u_{2}+2 u_{3}\right)+\boldsymbol{R}_{\geq 0}\left(u_{1}+2 u_{2}+2 u_{3}\right)+\boldsymbol{R}_{\geq 0}\left(2 u_{1}+u_{2}+2 u_{3}\right)$. Then $(Y, y)$ is 2-Gorenstein and $v_{0}=(1 / 2) v_{3}$ satisfies the condition of Proposition 1.2. We see that $\sigma^{*} \cap M$ is generated by $l_{1}=-2 v_{1}-2 v_{2}+3 v_{3}, l_{2}=-v_{1}+v_{3}, l_{3}=-2 v_{1}+$ $2 v_{2}+v_{3}, \quad l_{4}=v_{2}, \quad l_{5}=2 v_{1}+2 v_{2}-v_{3}, \quad l_{6}=v_{1}, \quad l_{7}=2 v_{1}-2 v_{2}+v_{3}$ and $l_{8}=-v_{2}+v_{3}$. (See Figure 2.) Let $z_{2}=\boldsymbol{e}\left(l_{2}\right)$ for $i=1$ through 8 and let $f=z_{2}-z_{4}+z_{6}+z_{8}$. Then $f$ is non-degenerate, $(X, x)$ is an isolated singularity and $X \wedge \operatorname{Sing}(Y)=\{x\}$. Moreover, $(X, x)$ is a quotient of a simple elliptic singularity.

Example 3. Let $n=3$, let $\left\{u_{1}, u_{2}, u_{3}, u_{4}\right\}$ be a basis of $N$ and let $\left\{v_{1}, v_{2}, v_{3}, v_{4}\right\}$ be the basis of $M$ dual to $\left\{u_{1}, u_{2}, u_{3}, u_{4}\right\}$. Let $\sigma=\boldsymbol{R}_{\geq 0}\left(u_{1}+u_{2}+2 u_{4}\right)+\boldsymbol{R}_{\geq 0}\left(u_{1}+u_{3}\right.$ $\left.+2 u_{4}\right)+\boldsymbol{R}_{\geq 0}\left(u_{2}+u_{3}+2 u_{4}\right)+\boldsymbol{R}_{\geq 0}\left(u_{1}+u_{2}+2 u_{3}+2 u_{4}\right)+\boldsymbol{R}_{\geq 0}\left(u_{1}+2 u_{2}+u_{3}+2 u_{4}\right)+\boldsymbol{R}_{\geq 0}\left(2 u_{1}\right.$ $\left.+u_{2}+u_{3}+2 u_{4}\right)$. Then $(Y, y)$ is 2 -Gorenstein and $v_{0}=(1 / 2) v_{4}$ satisfies the condition of Proposition 1.2. We see that $\sigma^{*} \cap M$ is generated by $l_{1}=v_{1}-v_{2}-v_{3}+v_{4}$, $l_{2}=v_{1}-v_{2}+v_{3}, \quad l_{3}=v_{1}+v_{2}+v_{3}-v_{4}, \quad l_{4}=v_{1}+v_{2}-v_{3}, \quad l_{5}=-v_{1}-v_{2}-v_{3}+2 v_{4}, \quad l_{6}=-v_{1}-v_{2}$ $+v_{3}+v_{4}, l_{7}=-v_{1}+v_{2}+v_{3}, l_{8}=-v_{1}+v_{2}-v_{3}+v_{4}, l_{9}=v_{1}, l_{10}=-v_{2}+v_{4}, l_{11}=v_{3}, l_{12}=v_{2}$, $l_{13}=-v_{3}+v_{4}$ and $l_{14}=-v_{1}+v_{4}$. (See Figure 3.) Let $z_{\imath}=\boldsymbol{e}\left(l_{\imath}\right)$, for $\imath=1$ through 14 and let $f=\sum_{1 \leqq \imath \leqq 14} z_{\imath}$. Then $f$ is non-degenerate, $(X, x)$ is an isolated singularity 

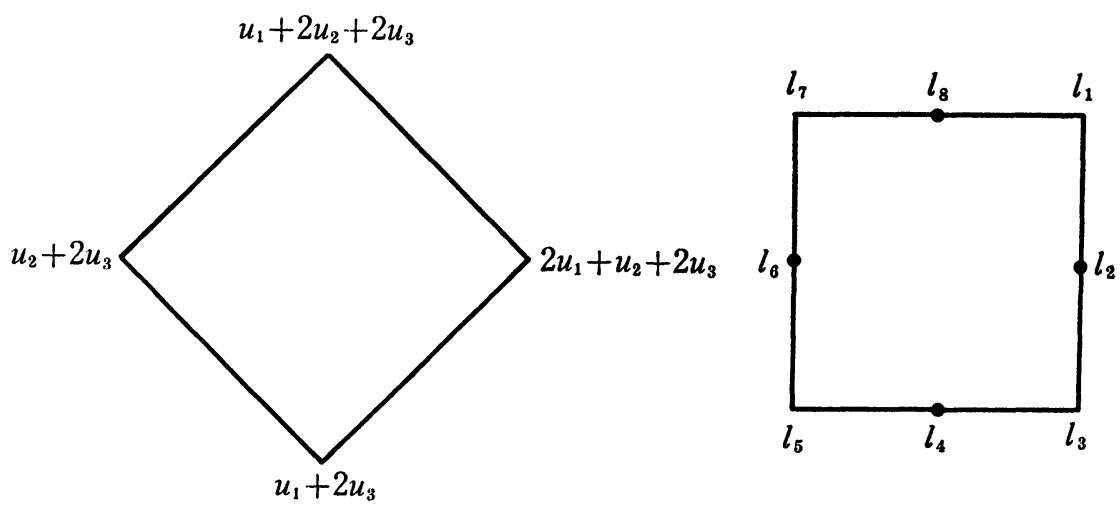

Figure 2.
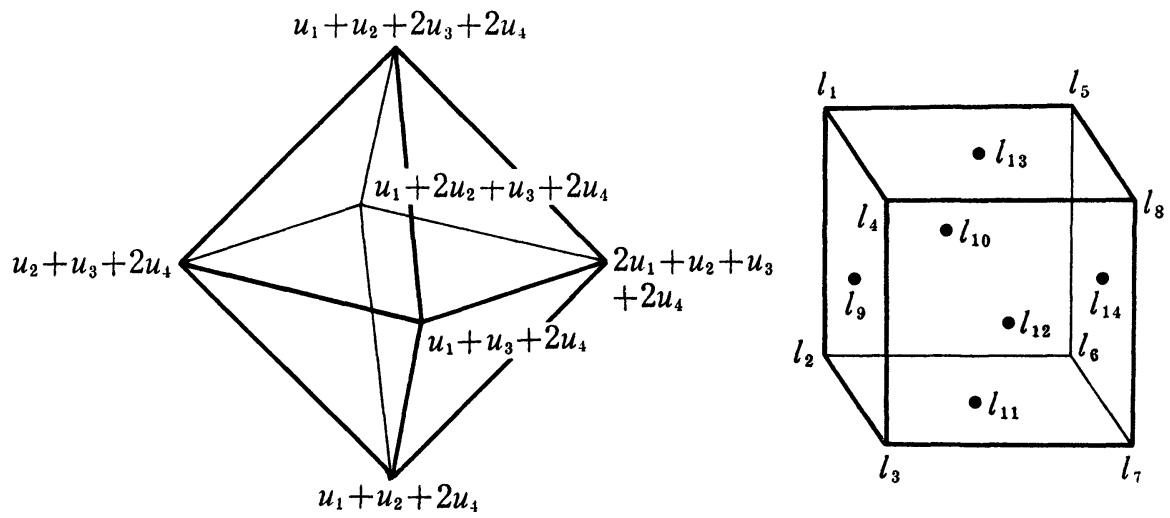

Figure 3.

and $X \cap \operatorname{Sing}(Y)=\{x\}$. Let $\Sigma=\left\{\right.$ faces of $\boldsymbol{R}_{z_{0}}\left(u_{1}+u_{2}+u_{3}+2 u_{4}\right)+\tau \mid \tau$ are 3-dimensional faces of $\sigma\}$ and let $\tilde{Y}=T_{N} \operatorname{emb}(\Sigma)$. Then $\Sigma=\Gamma^{*}(f)$ and $\tilde{Y}$ is the blowing up of $Y$ along $y=\operatorname{orb}(\sigma)$. Although $\tilde{Y}$ has singularities, $\tilde{X} \cap \operatorname{Sing}(\tilde{Y})=\phi$, where $\tilde{X}$ is the proper transformation of $X$ under the blowing up $\Pi: \tilde{Y} \rightarrow Y$. Moreover, $\Pi^{-1}(y) \cap \tilde{X}=E\left(u_{1}+u_{2}+u_{3}+2 u_{4}\right)$ is an Enriques surface. Each of small deformations $X_{\varepsilon}=\{f=\varepsilon\}$ of $X$ has eight isolated quotient singularities.

\section{§ 3. Hyperplane sections of Gorenstein toric singularities}

We keep the notations of the previous section and throughout this section, we assume that $(Y, y)$ is an isolated (, i.e., each $n$-dimensional face of $\sigma$ is non-singular), non-terminal and Gorenstein singularity. Hence $(Y, y)$ is a canonical singularity of index 1 and the set $\mathcal{L}:=\left\{u \in \operatorname{Int}(\sigma) \cap N \mid\left\langle v_{0}, u\right\rangle=1\right\}$ is non- 
empty. Moreover, we assume that $X=\{f=0\}$ is a generic hyperplane section, i.e., $f=\Sigma_{c_{v}} e(v)$ with $c_{v} \neq 0$, for the generators $v$ of $\sigma^{*} \cap M$.

Proposition 3.1. Under the above assumptions, $(X, x)$ is a purely elliptic singularity, i.e., $\delta_{m}(X, x)=1$ for each positive integer $m$.

Proof. Let $u_{0}$ be an element in $\mathcal{L}$. Then $\left\langle v_{0}, u_{0}\right\rangle=1$ and $\left\{v \in \sigma^{*} \mid\left\langle v, u_{0}\right\rangle\right.$ $\geqq 1\} \supset$ the convex hull of $\left(\sigma^{*} \backslash\{0\}\right) \cap M=\Gamma_{+}(f) \ni v_{0}$. Hence the set $\left\{v \in \sigma^{*} \mid\left\langle v, u_{0}\right\rangle\right.$ $=1\} \cap \Gamma_{+}(f)$ is a compact face of $\Gamma_{+}(f)$ and contains $v_{0}$. Therefore, $\delta_{m}=1$ for each positive integer $m$, by Theorem 2.2 .

q.e.d.

Remark. (1) If $(Y, y)$ is non-terminal and canonical of index $r>1$, then $(Y, y)$ is $r$-Gorenstein and $v_{0} \in \sigma^{*} \backslash \operatorname{Int}\left(\Gamma_{+}(f)\right)$. There are examples with $v_{0} \notin$ $\Gamma_{+}(f)$, as well as examples with $v_{0} \in \Gamma(f)$. Hence $(X, x)$ may not be a periodically elliptic singularity in contrast with the above proposition.

(2) In the case that $(Y, y)$ is not isolated, if $\mathcal{L}=\phi$, then $(X, x)$ may be an isolated canonical singularity, even though $(Y, y)$ is a non-terminal Gorenstein singularity. For instance, let $\sigma$ be the cone generated by $( \pm 1,0,0,1),(0, \pm 1,0,1)$ and $(1,1,2,1)$ in $\boldsymbol{Z}^{4}$.

Ishii [3] and Koyama independently showed that a 2-dimensional purely elliptic singularity is a simple elliptic singularity or a cusp singularity.

PROPOSITION 3.2. When $n=2,(X, x)$ is a simple elliptic singularity (resp. a cusp singularity), if the cardinal number of $\mathcal{L}$ is equal to (resp. greater than) 1.

Proof. First, we consider the case that $\mathcal{L}$ consists of one element $u_{0}$. For each 2-dimensional face $\tau=\boldsymbol{R}_{z_{0}} u^{\prime}+\boldsymbol{R}_{\geq_{0} 0} u^{\prime \prime}\left(\left\{u^{\prime}, u^{\prime \prime}\right\} \subset\left\{u_{1}, u_{2}, \cdots, u_{s}\right\}\right)$ of $\sigma$, $\left\{u_{0}, u^{\prime}, u^{\prime \prime}\right\}$ is a basis of $N$, because $\left\langle v_{0}, u_{0}\right\rangle=\left\langle v_{0}, u^{\prime}\right\rangle=\left\langle v_{0}, u^{\prime \prime}\right\rangle=1$ and the triangle spanned by $u_{0}, u^{\prime}$ and $u^{\prime \prime}$ contains no elements in $N$ except $u_{0}, u^{\prime}$ and $u^{\prime \prime}$. Let $\left\{v_{\tau}, v^{\prime}, v^{\prime \prime}\right\}$ be the basis of $M$ dual to $\left\{u_{0}, u^{\prime}, u^{\prime \prime}\right\}$. Then $\left\langle v_{\tau}, u_{0}\right\rangle=1$ and $\left\langle v_{\tau}, u^{\prime}\right\rangle=\left\langle v_{\tau}, u^{\prime \prime}\right\rangle=0$. Hence $\sigma^{*}$ is generated by $v_{\tau}$ and $\Gamma(f)$ consists of one face which is the polygon spanned by $v_{\tau}$, for all 2-dimensional faces $\tau$ of $\sigma$. Therefore, $\Gamma^{*}(f)=\left\{\right.$ faces of $\boldsymbol{R}_{\geq 0} u_{0}+\tau \mid \tau$ are 2-dimensional faces of $\left.\sigma\right\}$ and the exceptional set $E=E\left(u_{0}\right)$ of the resolution of $(X, x)$ obtained from $\Gamma^{*}(f)$ is a non-singular curve. It should be elliptic, because $\delta_{m}(X, x)=1$.

When the cardinal number of $\mathcal{L}$ is greater than 1 , we easily see that there exist at least two 2-dimensional compact faces of $\Gamma_{+}(f)$ containing $v_{0}$, which we denote by $\Delta_{1}$ and $\Delta_{2}$. Then $\Delta_{1}^{*}=\boldsymbol{R}_{z_{0}} u_{1}^{\prime}$ and $\Delta_{2}^{*}=\boldsymbol{R}_{\geq_{0}} u_{2}^{\prime}$ for primitive elements $u_{1}^{\prime}$ and $u_{2}^{\prime}$ in $\operatorname{Int}(\sigma) \cap N$ such that $\left\langle v_{0}, u_{1}^{\prime}\right\rangle=d\left(u_{1}^{\prime}\right)$ and that $\left\langle v_{0}, u_{2}^{\prime}\right\rangle=d\left(u_{2}^{\prime}\right)$. Hence the exceptional set $E$ of the resolution $\pi:(\tilde{X}, E) \rightarrow(X, x)$ of $(X, x)$ obtained from any subdivision of $\Gamma^{*}(f)$ contains two irreducible components $E\left(u_{1}^{\prime}\right)$ and $E\left(u_{2}^{\prime}\right)$ along which $\pi * \omega$ has poles of order 1 , by Lemma 2.3 , where $\omega=\operatorname{Res}\left(\boldsymbol{e}\left(v_{0}\right)\left(\left(d w_{1} / w_{1}\right)\right.\right.$ $\left.\left.\wedge \cdots \wedge\left(d w_{n+1} / w_{n+1}\right)\right) / f\right)$. Therefore, $(X, x)$ is not a simple elliptic singularity. 
Since $(Y, y)$ is an isolated singularity, $(X, x)$ is smoothable. On the other hand, Wahl $[9,10]$ showed that if a simple elliptic singularity (resp. a cusp singularity) $(X, x)$ is smoothable, then $m(X) \leqq 9$, (resp. $m(X)-l(X) \leqq 9)$, where $l(X)$ is the number of the irreducible components of the exceptional set $E$ of the minimal resolution of $(X, x)$ and $m(X)$ is the multiplicity of $(X, x)$, which is equal to $-E^{2}$, if $-E^{2} \geqq 3$.

Proposition 3.3. Assume that the cardinal number of $\mathcal{L}$ is equal to 1 . If $\sigma$ is an s-gonal cone, $-E^{2}=12-s$. (Therefore, $-E^{2} \leqq 9$.)

Proof. Let $\mathcal{L}=\left\{u_{0}\right\}$. Then $\Gamma^{*}(f)=\left\{\right.$ faces of $\boldsymbol{R}_{\geq 0} u_{0}+\tau \mid \tau$ are 2-dimensional faces of $\sigma\}$ consists of non-singular cones, by the proof of Proposition 3.2. Hence we obtain resolutions $\Pi:(\tilde{Y}, F) \rightarrow(Y, y)$ and $\pi=\Pi_{1 \tilde{X}}:(\tilde{X}, E) \rightarrow(X, x)$, where $\tilde{Y}=T_{N} \operatorname{emb}\left(\Gamma^{*}(f)\right), F$ is the closure of $\operatorname{orb}\left(\boldsymbol{R}_{\geq 0} u_{0}\right), \tilde{X}$ is the proper transformation of $X$ under $\Pi$ and $E=\tilde{X} \cdot F$. Let $\widetilde{D}_{\imath}$ be the proper transformation of $D_{i}$ under $\Pi$ and let $E_{\imath}=F \cdot \widetilde{D}_{\imath}$. Since $F+\tilde{X}=[\Pi * f]$ and $F+\widetilde{D}_{1}+\widetilde{D}_{2}+\cdots+\widetilde{D}_{s}$ $=\left[\Pi * \boldsymbol{e}\left(v_{0}\right)\right]$ are principal divisors, we have $-E_{i \tilde{X}}^{2}=-F^{2} \cdot \tilde{X}=F \cdot \tilde{X}^{2}=\sum_{1 \leqq \imath \leqq s} F \cdot \widetilde{D}_{\imath}^{2}$ $+2 \Sigma_{0 \leqq i<\jmath \leqq s} F \cdot \widetilde{D}_{i} \cdot \widetilde{D}_{\jmath}=\left(\Sigma_{1 \leqq i \leqq s} E_{i 1 F}^{2}\right)+2 s=3(4-s)+2 s=12-s$, because $F$ is a nonsingular toric variety whose 1 -dimensional orbits are $E_{1}, E_{2}, \cdots$ and $E_{s}$.

q.e.d.

Proposition 3.4. Assume that the convex hull of $\mathcal{L}$ is a polygon. If $\sigma$ is an s-gonal cone, then, $-E^{2}-l(X)=12-s$. (Therefore, $-E^{2}-l(X) \leqq 9$.)

Proof. Let $P$ (resp. $Q$ ) be the convex hull of $\mathcal{L}$ (resp. $\left\{u \in \sigma \cap N \mid\left\langle v_{0}, u\right\rangle=\right.$ 1\}). Then $Q=\left\{u \in \sigma \mid\left\langle v_{0}, u\right\rangle=1\right\}$ and $\operatorname{Int}(Q) \supset P$. Take a triangulation $\Delta$ (resp. $\Delta^{\prime}$ ) of $P$ (resp. $Q \backslash \operatorname{Int}(P)$ ) so that the set of the vertices of $\Delta$ (resp. $\Delta^{\prime}$ ) agrees with $P \cap N=\mathcal{L}$ (resp. $(Q \backslash \operatorname{Int}(P)) \cap N)$. Let $e_{0}, e_{1}$ and $e_{2}$ (resp. $e_{0}^{\prime}, e_{1}^{\prime}$ and $\left.e_{2}^{\prime}\right)$ be the numbers of the vertices, edges and faces, respectively, of $\Delta$ (resp. $\Delta^{\prime}$ ). Then $e_{0}-e_{1}+e_{2}=1$ and $e_{0}^{\prime}-e_{1}^{\prime}+e_{2}^{\prime}=0$, because $P$ and $Q$ are polygons. Let $l$ be the number of the vertices on the boundary $\partial P$ of $P$. Then $e_{0}^{\prime}=l+s$ and $3 e_{2}^{\prime}=$ $2 e_{1}^{\prime}-(l+s)$, because the number of the vertices (resp. edges) on the boundary of $Q \backslash \operatorname{Int}(P)$ is equal to $l+s$. Hence by an easy calculation, we have $e_{1}^{\prime}=2(l+s)$. Since $\square:=\Delta \cup \Delta^{\prime}$ is a triangulation of $Q$, we see that $\Sigma^{*}:=\left\{\boldsymbol{R}_{\geq 0} \tau \mid \tau\right.$ are simplexes of $\square\} \cup\{0\}$ is a subdivision of $\Gamma^{*}(f)$ and consists of non-singular cones. Hence we have a resolution $\Pi:(\tilde{Y}, F) \rightarrow(Y, y)$, where $\tilde{Y}=T_{N}$ emb $\left(\Sigma^{*}\right)$. Let $\widetilde{D}_{\imath}$ be the proper transformation of $D_{\imath}$ under $\Pi$ and let $\widetilde{D}=\widetilde{D}_{1}+\widetilde{D}_{2}+\cdots+\widetilde{D}_{s}$. Then $\Delta$ and

are the dual graphs of $F=F_{1}+F_{2}+\cdots+F_{e_{0}}$ and $F+\tilde{D}$, respectively. Since $\widetilde{X}+F=\left[\Pi^{*} f\right]$ and $\widetilde{D}+F=\left[\Pi * e\left(v_{0}\right)\right]$ are principal divisors, we have $0=F_{i} \cdot F_{j}$ $\cdot(\tilde{D}+F)=F_{i}^{2} \cdot F_{j}+F_{i} \cdot F_{j}^{2}+2$, if $F_{i} \cap F_{j} \neq \phi$ and $-E_{1 \tilde{X}}^{2}=-F^{2} \cdot \tilde{X}=F \cdot \tilde{D}^{2}=\Sigma_{1 \leqq \imath \leqq e_{0}}$ $\left(\sum_{1 \leqq j \leqq s} F_{i} \cdot \widetilde{D}_{j}^{2}+2 \sum_{1 \leqq j<k \leqq s} F_{i} \cdot \widetilde{D}_{j} \cdot \widetilde{D}_{k}\right)=\sum_{1 \leqq \imath \leqq e_{0}, 1 \leqq \jmath \leqq s} F_{i} \cdot \widetilde{D}_{j}^{2}+2 s$, where $\tilde{X}$ is the proper transformation of $X$ under $\Pi$ and $E=\tilde{X} \cdot F$. On the other hand, since each irreducible component $F_{\imath}$ of $F$ is a non-singular toric variety with $F_{i} \cdot\left(F+\widetilde{D}-F_{\imath}\right)$ as the union of 1 -dimensional orbits, we have $\sum_{\imath \neq j} F_{i} \cdot F_{j}^{2}+\sum_{1 \leqq k \leqq s} F_{i} \cdot \widetilde{D}_{k}^{2}=3\left(4-d_{\imath}\right)$, where $d_{\imath}$ is the number of the double curves on $F_{\imath}$. Hence by taking the sum 
of the self-intersection numbers of the double curves $F_{i} \cdot F_{\jmath}$ and $F_{i} \cdot \widetilde{D}_{k}$ on all the irreducible components $F_{\imath}$ of $F$, we have $-2 e_{1}+\sum_{1 \leqq \imath \leqq e_{0}, 1 \leqq \jmath \leqq s} F_{i} \cdot \widetilde{D}_{\jmath}^{2}=\sum_{1 \leqq \imath \leqq e_{0}} 3(4-$ $\left.d_{\imath}\right)=12 e_{0}-3\left(2 e_{1}+l+s\right)=12 e_{0}-6 e_{1}-3 l-3 s$. Therefore, $-E_{\mid \tilde{X}}^{2}=12 e_{0}-6 e_{1}-3 l-3 s+$ $2 e_{1}+2 s=12 e_{0}-4 e_{1}-3 l-s=12 e_{0}-12 e_{1}+12 e_{2}+l-s=12+l-s$, because $3 e_{2}=2 e_{1}-l$. Thus we obtain $-E^{2}-l=12-s$. Here we note that $l$ is equal to the number of the irreducible components of $E$, because $\tilde{X} \cap F_{\imath} \neq \phi$, if and only if $\widetilde{D} \cap F_{\imath} \neq \phi$ (, i.e., the vertex of $\square$ corresponding to $F_{\imath}$ is on $\partial P$ ) and then $\tilde{X} \cap F_{\imath}$ is irreducible. Moreover, $E_{i} \cdot E_{\jmath}=\tilde{X} \cdot F_{i} \cdot F_{\jmath}=\tilde{D} \cdot F_{i} \cdot F_{\jmath} \leqq 1$ and the equality holds, if and only if the vertices of $\square$ corresponding to $F_{\imath}$ and $F$, are joined by an edge on $\partial P$. Hence $E$ forms a cycle. Therefore, although $(\tilde{X}, E)$ is not a minimal resolution, the contraction of a rational curve $E_{\imath}$ with $E_{\imath}^{2}=-1$ does not change the number $-E^{2}-l$. Thus we complete the proof.

Examples. In the following table, $E=E_{1}+E_{2}+\cdots+E_{l}$ is the exceptional set of the minimal resolution of $(X, x)$ such that $E_{i} \cdot E_{\imath+1}=1$ for each $i \in \boldsymbol{Z} / l \boldsymbol{Z}$.

\begin{tabular}{c|l|l} 
generators of $\sigma$ & $l$ & $-E_{1}^{2},-E_{2}^{2}, \cdots,-E_{l}^{2}$ \\
\hline$(0,0,1),(5,2,1),(3,5,1)$ & 6 & $5,4,5,4,5,4$ \\
$(0,0,1),(4,1,1),(3,4,1)$ & 6 & $7,2,7,2,7,2$ \\
$(0,0,1),(8,3,1),(5,8,1)$ & 9 & $5,4,3,5,4,3,5,4,3$ \\
$(0,0,1),(7,2,1),(5,7,1)$ & 9 & $5,5,2,5,5,2,5,5,2$ \\
$(0,0,1),(7,3,1),(4,7,1)$ & 9 & $6,4,2,6,4,2,6,4,2$
\end{tabular}

\section{$\S 4$. Deformations}

We assume that $n=\operatorname{dim} X \geqq 3$, throughout this section. Let $U=X \backslash\{x\}$ and let $W=Y \backslash\{y\}$. Then we have the isomorphism $T_{X} \cong H^{1}\left(U, \Theta_{U}\right)$, by Proposition 2.1 and [7, Theorem 2], where $T_{X}^{1}=H^{0}\left(X, \Theta_{X}^{1}\right)$ is the tangent space to the formal moduli space of $X$ and $\Theta_{U}$ is the tangent sheaf of $U$. Consider the long exact sequence arising from the short exact sequence of sheaves:

$$
0 \longrightarrow \Theta_{U} \longrightarrow i * \Theta_{W} \longrightarrow \Re \longrightarrow 0,
$$

where $i: U \hookrightarrow W$ is the inclusion map. Here we note that the normal sheaf $\Re \cong$ $\mathcal{O}_{U}(U)$ is isomorphic to the structure sheaf $\mathcal{O}_{U}$, because $X$ is a principal divisor on $\cdot Y$. Let $\left\{\theta_{1}, \theta_{2}, \cdots, \theta_{l}\right\}$ be a basis of the image of the map $\delta: H^{0}(U, \Re) \rightarrow$ $H^{1}\left(U, \Theta_{U}\right)$ and let $g_{\imath}$ be an element of $H^{0}\left(Y, \mathcal{O}_{Y}\right)$ whose image is $\theta_{\imath}$ under the composite of the surjective maps $H^{0}\left(Y, \mathcal{O}_{Y}\right)=H^{0}\left(W, \mathcal{O}_{W}\right) \rightarrow H^{0}\left(U, \mathcal{O}_{U}\right) \cong H^{0}(U, \Re)$ sending $h$ to $h_{\mid U} \cdot \partial / \partial f$ and $H^{0}(U, \Re) \rightarrow \operatorname{Im}(\delta)$. Let $\mathscr{X}=\left\{(z, t) \in Y \times \Delta \mid f(z)+t_{1} g_{1}(z)\right.$ $\left.+t_{2} g_{2}(z)+\cdots+t_{l} g_{l}(z)=0\right\}$ and let $\pi$ be the restriction to $\mathscr{X}$ of the projection 
$Y \times \Delta \rightarrow \Delta$, where $\Delta=\left\{\left(t_{1}, t_{2}, \cdots, t_{l}\right) \in C^{l}|| t_{j} \mid<\varepsilon\right\}$. Then $\pi$ is flat, by [1, Chapter $\mathrm{V}$, Corollary 1.5]. Let $\mathcal{U}$ be the open set of $\mathscr{X}$ on which $\pi$ is smooth. Then we obtain a family $\pi_{1}: U \rightarrow \Delta$ of deformations of the complex manifold $U$. Moreover, by an easy calculation, we have $\rho\left(\partial / \partial t_{j}\right)=\theta$, for $j=1$ through $l$, where $\rho: T_{0}(\Delta) \rightarrow H^{1}\left(U, \Theta_{U}\right)$ is the infinitesimal deformation map. Hence $\rho$ is injective and if $H^{1}\left(U, i * \Theta_{W}\right)=0$, then $\rho$ is surjective.

THEOREM 4.1. If $H^{1}\left(U, i^{*} \Theta_{W}\right)=0$, then $\pi: X \rightarrow \Delta$ is a locally semiuniversal family of $X$.

Proof. Recall that $T_{X}^{1}$ is defined by the exact sequence

$$
0 \longrightarrow \operatorname{Hom}\left(\Omega_{X}^{1}, \mathcal{O}_{X}\right) \longrightarrow \operatorname{Hom}\left(j^{*} \Omega_{\boldsymbol{C}^{N}}, \mathcal{O}_{X}\right) \longrightarrow \operatorname{Hom}\left(I / I^{2}, \mathcal{O}_{X}\right) \longrightarrow T_{X}^{1} \longrightarrow 0
$$

obtained by the exact sequence of sheaves: $I / I^{2} \stackrel{d}{\rightarrow} j^{*} \Omega_{c^{N}} \rightarrow \Omega_{X}^{1} \rightarrow 0$, for an inclusion $j: X \hookrightarrow_{C} C^{N}$ with the ideal sheaf $I$. On the other hand, we have the exact sequence

$$
\begin{aligned}
0 \longrightarrow \operatorname{Hom}\left(\Omega_{X}^{1}, \mathcal{O}_{X}\right) \longrightarrow \operatorname{Hom}\left(j^{*} \Omega_{c^{N}}^{1}, \mathcal{O}_{X}\right) \longrightarrow \operatorname{Hom}\left(\operatorname{Im}(d), \mathcal{O}_{X}\right) \\
\longrightarrow \operatorname{Ext}_{\mathcal{O}_{X}}^{1}\left(\Omega_{X}^{1}, \mathcal{O}_{X}\right) \longrightarrow 0
\end{aligned}
$$

by the short exact sequence of sheaves: $0 \rightarrow \operatorname{Im}(d) \rightarrow j * \Omega_{c^{N}} \rightarrow \Omega_{X}^{1} \rightarrow 0$. Since the support of $\operatorname{ker}(d)$ is $\{x\}$, we have $\operatorname{Hom}\left(\operatorname{Im}(d), \mathcal{O}_{X}\right)=\operatorname{Hom}\left(I / I^{2}, \mathcal{O}_{X}\right)$. Thus we have the canonical isomorphism $\operatorname{Ext}_{\mathcal{O}_{X}}^{1}\left(\Omega_{X}^{1}, \mathcal{O}_{X}\right) \cong T_{X}^{1}$. Hence the infinitesimal deformation map $T_{0}(\Delta) \rightarrow \operatorname{Ext}_{O_{X}}^{1}\left(\Omega_{X}^{1}, O_{X}\right)$ for the family $\pi: \mathscr{X} \rightarrow \Delta$ is bijective. Then by $[8$, Theorem 6.1$], \pi: \mathscr{X} \rightarrow \Delta$ is locally semiuniversal.

q.e.d.

COROLlary 4.2. If $H^{1}\left(U, i^{*} \Theta_{W}\right)=0$, then any small deformation of $X$ is also a hypersurface section of $Y$.

Proposition 4.3. If $\sigma$ is a simplicial cone (hence $Y$ is a quotient space of $\left.\boldsymbol{C}^{n+1}\right)$, then $H^{1}\left(U, i^{*} \Theta_{W}\right)=0$.

Proof. Let $l_{1}, l_{2}, \cdots$ and $l_{n+1}$ be the generators of $\sigma$ and let $N^{\prime}=\boldsymbol{Z} l_{1}+\boldsymbol{Z} l_{2}$ $+\cdots+\boldsymbol{Z} l_{n+1}$. Here we may assume that $l_{1}, l_{2}, \cdots$ and $l_{n+1}$ are primitive elements in $N$. Then the inclusion $N^{\prime} \hookrightarrow N$ induces a holomorphic map $\varphi: Y^{\prime} \rightarrow Y$, where $Y^{\prime}=T_{N^{\prime}}$ emb $(\{$ faces of $\sigma\}) \cong C^{n+1}$. Let $U^{\prime}=\varphi^{-1}(U)$. Then $\varphi_{U^{\prime}}: U^{\prime} \rightarrow U$ is unramified, by the assumption $X \cap \operatorname{Sing}(Y)=\{x\}$. Hence $H^{1}\left(U, i * \Theta_{W}\right)=H^{1}\left(U^{\prime}, h^{*} \Theta_{Y^{\prime}}\right)^{G}$ $=0$, where $h: U^{\prime} \subset Y^{\prime}$ is the inclusion map and $G$ is the covering transformation group of $\varphi$.

q.e.d.

Example. Let $X^{\prime}$ be the hypersurface of $C^{4}$ defined by $z_{1}^{2}+z_{2}^{6}+z_{3}^{6}+z_{4}^{6}=0$ and let $X=X^{\prime} / G$ be the quotient space of $X^{\prime}$ under the group $G$ generated by $(1, \xi, \xi, \xi)$, where $\xi$ is a primitive cube root of 1 . Then $X$ is a hypersurface section of $Y=C^{4} / G$, which is a toric singularity, and whose singular locus 
Sing $(Y)$ is 1-dimensional. We easily see that $X$ has an isolated singularity obtained by contracting a K3 surface. By Corollary 4.2 and Proposition 4.3, any small deformation of $X$ is also a hypersurface section $X_{t}=\pi^{-1}(t)$ of $Y$. Since $X_{t}$ intersect $\operatorname{Sing}(Y)$ at finitely many points, $X_{t}$ has singularities, i.e., $X$ is not smoothable.

\section{REFERENCES}

[1] C. BăNiCA AND O. STĂNĂȘILĂ, Algebraic methods in the global theory of complex spaces, Editula Academiei, București and John Wiley \& Sons, London New York, Sydney and Tronto, 1976.

[2] S. IsHiI, Isolated $\boldsymbol{Q}$-Gorenstein singularities of dimension three, Complex analytic singularities (T. Suwa and P. Wagreigh, eds.), Advanced Studies in Pure Math. 8, Kinokuniya, Tokyo and North-Holland, Amsterdam, New York, Oxford, 1986, $165-198$.

[3] S. IsHII, Two dimensional singularities with bounded plurigenera $\delta_{m}$ are $\boldsymbol{Q}$. Gorenstein singularities, to appear in Proc. Iowa city singularities conference, Contemporary Mathematics series of AMS.

[4] T. ODA, Convex Bodies and Algebraic Geometry, Ergebnisse der Mathematik und ihrer Grenzgebiete 3. Folge·Band 15, Springer-Verlag, Berlin, Heidelberg, New York, London, Paris, Tokyo, 1987.

[5] М. ОкA, On the resolution of the hypersurface singularities, Complex analytic singularities (T. Suwa and P. Wagreigh, eds.), Advanced Studies in Pure Math. 8, Kinokuniya, Tokyo and North-Holland, Amsterdam, New York, Oxford, 1986, 405-436.

[6] M. ReID, Canonical 3-folds, in Journées de Géomérie Algébrique d’Angers, 1979 (A. Beauville, ed.). Sijthoff \& Noordhoff, Alphen aan den Rijn, The Netherlands and Rockville, Md USA, 1980, 273-310.

[7] M. Schlessinger, Rigidity of quotient singularities, Inventiones Math. 14 (1971), $17-26$.

[8] G. N. TJURINA, Locally semiuniversal flat deformations of isolated singularities of complex spaces. Izv. Akad. Nauk SSSR, ser. Mat. Tom 33, No. 5 (1970) (In Russian).

[9] J. WAHL, Elliptic deformations of minimally elliptic singularities, Math. Ann. 253 (1980), 241-262.

[10] J. WAHL, Smoothings of normal surface singularities, Topology 20 (1981), 219246.

[11] K. Watanabe, On plurigenera of normal isolated singularities II, Complex analytic singularities (T. Suwa and P. Wagreigh, eds.), Advanced Studies in Pure Math. 8, Kinokuniya, Tokyo and North-Holland, Amsterdam, New York, Oxford, 1986, 671-685.

Faculty of Liberal ARts

TÔHOKU GAKUIN UNIVERSiTY

SENDAI, 981-31

JAPAN 